\title{
REALIZATIONS OF INNER AUTOMORPHISMS OF ORDER FOUR AND FIXED POINTS SUBGROUPS BY THEM ON THE CONNECTED COMPACT EXCEPTIONAL LIE GROUP $E_{8}$, PART II
}

BY

\author{
TOSHIKAZU MIYASHITA
}

\begin{abstract}
AвstRact. The compact simply connected Riemannian 4-symmetric spaces were classified by J.A. Jiménez according to type of the Lie algebras. As homogeneous manifolds, these spaces are of the form $G / H$, where $G$ is a connected compact simple Lie group with an automorphism $\tilde{\gamma}$ of order four on $G$ and $H$ is a fixed points subgroup $G^{\gamma}$ of $G$. According to the classification by J.A. Jiménez, there exist seven compact simply connected Riemannian 4-symmetric spaces $G / H$ in the case where $G$ is of type $E_{8}$. In the present article, we give the explicit form of automorphisms $\tilde{w}_{4} \tilde{v}_{4}$ and $\tilde{\mu}_{4}$ of order four on $E_{8}$ induced by the $C$-linear transformations $w_{4}, v_{4}$ and $\mu_{4}$ of the 248-dimensional vector space $e_{8} C$, respectively. Further, we determine the structure of these fixed points subgroups $\left(E_{8}\right)^{w_{4}},\left(E_{8}\right)^{v_{4}}$ and $\left(E_{8}\right)^{\mu_{4}}$ of $E_{8}$. These amount to the global realizations of three spaces among seven Riemannian 4-symmetric spaces $G / H$ above corresponding to the Lie algebras $\mathfrak{h}=i \boldsymbol{R} \oplus \mathfrak{s u}(8), i \boldsymbol{R} \oplus \mathfrak{e}_{7}$ and $\mathfrak{h}=\mathfrak{s u}(2) \oplus \mathfrak{s u}(8)$, where $\mathfrak{h}=\operatorname{Lie}(H)$.
\end{abstract}

\section{INTRODUCTION}

Let $G$ be a Lie group and $H$ a compact subgroup of $G$. A homogeneous space $G / H$ with $G$-invariant Riemannian metric $g$ is called a Riemannian 4-symmetric space if there exists an automorphism $\tilde{\gamma}$ of order four on $G$ such that $\left(G^{\gamma}\right)_{0} \subset H \subset G^{\gamma}$, where $G^{\gamma}$ and $\left(G^{\gamma}\right)_{0}$ are the fixed points subgroup of $G$ by $\tilde{\gamma}$ and its identity component, respectively.

Now, for the exceptional compact Lie group of type $E_{8}$, as in Table below, there exist seven cases of the compact simply connected Riemannian 4-symmetric spaces which were classified by J.A. Jiménez as mentioned in abstract ([3]). Accordingly, our interest is to realize the groupfication for the classification as Lie algebra.

Our results of groupfication corresponding to the Lie algebra $\mathfrak{h}$ in Table are given as follows.

\begin{tabular}{|c|c|c|c|}
\hline Case & $\mathfrak{h}$ & $\tilde{\gamma}$ & $H=G^{\gamma}$ \\
\hline
\end{tabular}

2010 Mathematics Subject Classification. 53C30, 53C35, 17B40.

Key words. 4-symmetric spaces, exceptional Lie groups. 


$$
\begin{array}{llll}
2 & i \boldsymbol{R} \oplus \mathfrak{s u}(8) & \tilde{w}_{4} & (U(1) \times S U(8)) / \boldsymbol{Z}_{24} \\
3 & i \boldsymbol{R} \oplus \mathfrak{e}_{7} & \tilde{v}_{4} & \left(U(1) \times E_{7}\right) / \boldsymbol{Z}_{2} \\
4 & \mathfrak{s u}(2) \oplus \mathfrak{s u}(8) & \tilde{\mu}_{4} & (S U(2) \times S U(8)) / \boldsymbol{Z}_{4} \\
5 & \mathfrak{s u}(2) \oplus i \boldsymbol{R} \oplus \mathfrak{e}_{6} & \tilde{\omega}_{4} & \left(S U(2) \times U(1) \times E_{6}\right) /\left(\boldsymbol{Z}_{2} \times \boldsymbol{Z}_{3}\right) \\
6 & i \boldsymbol{R} \oplus \mathfrak{s v}(14) & \tilde{\kappa}_{4} & (U(1) \times \operatorname{Spin}(14)) / \boldsymbol{Z}_{4} \\
7 & \mathfrak{s u}(2) \oplus i \boldsymbol{R} \oplus \mathfrak{s p}(12) & \tilde{\varepsilon}_{4} & (S U(2) \times U(1) \times \operatorname{Spin}(12)) /\left(\boldsymbol{Z}_{2} \times \boldsymbol{Z}_{2}\right)
\end{array}
$$

In [5], the author showed the group realizations for Case 1 in Table. In the present article, we state the realizations of the group $H$ for Case 2,3 and 4. The remaining cases will be shown in a forthcoming article [6] by the author.

Finally, the author would like to say that the feature of this article is to give elementary proofs of the isomorphism of groups by using the homomorphism theorem except several proofs, and of the connectedness of groups as topological spaces.

This article is a continuation of [5], hence we start from Section 4. We refer the reader to [5] for preliminary results and also to [4], [5], [7], [9] or [10] for notations. Note that we change the numbering of Case 5 and Case 6 in [5] to the numbering of Case 3 and Case 4 in the present article, respectively.

\section{Case 2. The automorphism $\tilde{w}_{4}$ Of ORder four AND The group $\left(E_{8}\right)^{w_{4}}$}

In this case, we will study the connected compact exceptional Lie group of type $E_{8}$ constructed by S. Gomyo ([2]). With reference to [2], we rewrite its contents as detailed as possible. In particular, we give some proofs of lemma and theorem in which the proofs are omitted in [2].

First, in order to construct another $C$-Lie algebra of type $E_{8}$, we investigate the properties of the exterior $C$-vector space $\Lambda^{3}\left(C^{9}\right)$. Let $\boldsymbol{e}_{1}, \cdots, \boldsymbol{e}_{9}$ be the canonical $C$ basis of the nine dimensional $C$-vector space $C^{9}$ and $(\boldsymbol{x}, \boldsymbol{y})$ the inner product in $C^{9}$ satisfying $\left(\boldsymbol{e}_{i}, \boldsymbol{e}_{j}\right)=\delta_{i j}$, where $\delta_{i j}$ means the Kronecker's delta. In $\Lambda^{3}\left(C^{9}\right)$, we define an inner product by

$$
\begin{gathered}
\left(\boldsymbol{u}_{1} \wedge \boldsymbol{u}_{2} \wedge \boldsymbol{u}_{3}, \boldsymbol{v}_{1} \wedge \boldsymbol{v}_{2} \wedge \boldsymbol{v}_{3}\right)=\operatorname{det}\left(\left(\boldsymbol{u}_{i}, \boldsymbol{v}_{j}\right)\right) \\
(a, b)=a b, a, b \in \Lambda^{0}\left(C^{9}\right)=C .
\end{gathered}
$$

Here, $\boldsymbol{e}_{i_{1}} \wedge \boldsymbol{e}_{i_{2}} \wedge \boldsymbol{e}_{i_{3}}, i_{1}<i_{2}<i_{3}$ forms an orthonormal $C$-basis of $\Lambda^{3}\left(C^{9}\right)$. For $\boldsymbol{u} \in \Lambda^{3}\left(C^{9}\right)$, we define an element $* \boldsymbol{u} \in \Lambda^{6}\left(C^{9}\right)$ by

$$
(* u, v)=\left(u \wedge v, e_{1} \wedge \cdots \wedge e_{9}\right), v \in \Lambda^{6}\left(C^{9}\right) .
$$

Note that the inner product $(* \boldsymbol{u}, \boldsymbol{v})$ is defined as in the case $\Lambda^{3}\left(C^{9}\right)$ above. Then, $*$ induces a $C$-linear isomorphism

$$
*: \Lambda^{3}\left(C^{9}\right) \rightarrow \Lambda^{6}\left(C^{9}\right)
$$


and satisfies the following identity

$$
*^{2} \boldsymbol{u}=\boldsymbol{u}, \boldsymbol{u} \in \Lambda^{3}\left(C^{6}\right) .
$$

The Lie algebra $\mathfrak{s l}(9, C)$ of the group $S L(9, C)$ acts on $\Lambda^{3}\left(C^{9}\right)$ as follows:

$$
\begin{aligned}
& D\left(\boldsymbol{u}_{1} \wedge \boldsymbol{u}_{2} \wedge \boldsymbol{u}_{3}\right)=D \boldsymbol{u}_{1} \wedge \boldsymbol{u}_{2} \wedge \boldsymbol{u}_{3}+\boldsymbol{u}_{1} \wedge D \boldsymbol{u}_{2} \wedge \boldsymbol{u}_{3}+\boldsymbol{u}_{1} \wedge \boldsymbol{u}_{2} \wedge D \boldsymbol{u}_{3} \\
& D(1)=0, D \in \mathfrak{s l}(9, C)
\end{aligned}
$$

Lemma 4.1 ([2, Lemma 1.1]). For $D \in \mathfrak{s l}(9, C)$ and $\boldsymbol{u}, \boldsymbol{v} \in \Lambda^{3}\left(C^{9}\right)$, we have the following relational formulas

$$
(D \boldsymbol{u}, \boldsymbol{v})=\left(\boldsymbol{u},{ }^{t} D \boldsymbol{v}\right), *(D \boldsymbol{u})=-{ }^{t} D(* \boldsymbol{u}) .
$$

Proof. In order to prove this lemma, it is sufficient to prove these above for $\boldsymbol{u}=$ $u_{1} \wedge u_{2} \wedge u_{3}, v=v_{1} \wedge v_{2} \wedge v_{3} \in \Lambda^{3}\left(C^{9}\right)$. For the former formula, it follows that

$$
(D \boldsymbol{u}, \boldsymbol{v})=\operatorname{det}\left(\left(D \boldsymbol{u}_{i}, \boldsymbol{v}_{j}\right)\right)=\operatorname{det}\left(\left(\boldsymbol{u}_{i},{ }^{t} D \boldsymbol{v}_{j}\right)\right)=\left(\boldsymbol{u},{ }^{t} D \boldsymbol{v}\right) .
$$

For the latter formula, using the relational formula $D(\boldsymbol{u} \wedge \boldsymbol{w})=D \boldsymbol{u} \wedge \boldsymbol{w}+\boldsymbol{u} \wedge D \boldsymbol{w}$, we have the following

$$
\begin{aligned}
(*(D \boldsymbol{u}), \boldsymbol{w}) & =\left(D \boldsymbol{u} \wedge \boldsymbol{w}, \boldsymbol{e}_{1} \wedge \cdots \wedge \boldsymbol{e}_{9}\right) \\
& =\left(D(\boldsymbol{u} \wedge \boldsymbol{w})-\boldsymbol{u} \wedge D \boldsymbol{w}, \boldsymbol{e}_{1} \wedge \cdots \wedge \boldsymbol{e}_{9}\right) \\
& =\left(D(\boldsymbol{u} \wedge \boldsymbol{w}), \boldsymbol{e}_{1} \wedge \cdots \wedge \boldsymbol{e}_{9}\right)-\left(\boldsymbol{u} \wedge D \boldsymbol{w}, \boldsymbol{e}_{1} \wedge \cdots \wedge \boldsymbol{e}_{9}\right) \\
& =\left(\boldsymbol{u} \wedge \boldsymbol{w},{ }^{t} D\left(\boldsymbol{e}_{1} \wedge \cdots \wedge \boldsymbol{e}_{9}\right)\right)-\left(\boldsymbol{u} \wedge D \boldsymbol{w}, \boldsymbol{e}_{1} \wedge \cdots \wedge \boldsymbol{e}_{9}\right) \\
& =\left(\boldsymbol{u} \wedge \boldsymbol{w}, \sum_{i=1}^{9} \boldsymbol{e}_{1} \wedge \cdots \wedge{ }^{t} D \boldsymbol{e}_{i} \wedge \cdots \wedge \boldsymbol{e}_{9}\right)-\left(\boldsymbol{u} \wedge D \boldsymbol{w}, \boldsymbol{e}_{1} \wedge \cdots \wedge \boldsymbol{e}_{9}\right) \\
& =\left(\boldsymbol{u} \wedge \boldsymbol{w}, \operatorname{tr}\left({ }^{t} D\right)\left(\boldsymbol{e}_{1} \wedge \cdots \wedge \boldsymbol{e}_{9}\right)\right)-\left(\boldsymbol{u} \wedge D \boldsymbol{w}, \boldsymbol{e}_{1} \wedge \cdots \wedge \boldsymbol{e}_{9}\right) \\
& =-\left(\boldsymbol{u} \wedge D \boldsymbol{w}, \boldsymbol{e}_{1} \wedge \cdots \wedge \boldsymbol{e}_{9}\right) \\
& =(-* \boldsymbol{u}, D \boldsymbol{w}) \\
& =-\left({ }^{t} D(* \boldsymbol{u}), \boldsymbol{w}\right)
\end{aligned}
$$

for every $\boldsymbol{w} \in \Lambda^{6}\left(C^{9}\right)$. Hence we have $*(D \boldsymbol{u})=-{ }^{t} D(* \boldsymbol{u})$.

For $\boldsymbol{u}, \boldsymbol{v} \in \Lambda^{3}\left(C^{9}\right)$, we define a $C$-linear transformation $\boldsymbol{u} \times \boldsymbol{v}$ of $C^{9}$ by

$$
(\boldsymbol{u} \times \boldsymbol{v}) \boldsymbol{x}=*(\boldsymbol{v} \wedge *(\boldsymbol{u} \wedge \boldsymbol{x}))+\frac{2}{3}(\boldsymbol{u}, v) x, x \in C^{9} .
$$

Then we have the following lemma.

Lemma $4.2([2$, p.597]). We have $\operatorname{tr}(\boldsymbol{u} \times \boldsymbol{v})=0$.

Proof. In order to prove this lemma, we have to show the following relational formula

$$
(*(v \wedge *(u \wedge x)), y)=-(x \wedge u, y \wedge v), x, y \in C^{9} .
$$


Indeed, it follows from $* \boldsymbol{u}=\boldsymbol{u}, \boldsymbol{u} \in \Lambda^{3}\left(C^{9}\right)$ that

$$
\begin{aligned}
(*(v \wedge *(u \wedge x)), \boldsymbol{y}) & =\left((v \wedge *(u \wedge \boldsymbol{x})) \wedge \boldsymbol{y}, \boldsymbol{e}_{1} \wedge \cdots \wedge \boldsymbol{e}_{9}\right) \\
& =-\left(*(u \wedge \boldsymbol{x}) \wedge(\boldsymbol{v} \wedge \boldsymbol{y}), \boldsymbol{e}_{1} \wedge \cdots \wedge \boldsymbol{e}_{9}\right) \\
& =-\left(*^{2}(\boldsymbol{u} \wedge \boldsymbol{x}), \boldsymbol{v} \wedge \boldsymbol{y}\right) \\
& =-(\boldsymbol{u} \wedge \boldsymbol{x}, \boldsymbol{v} \wedge \boldsymbol{y}) \\
& =-(\boldsymbol{x} \wedge \boldsymbol{u}, \boldsymbol{y} \wedge \boldsymbol{v}) .
\end{aligned}
$$

Using $\operatorname{tr}(\boldsymbol{u} \times \boldsymbol{v})=\sum_{i=1}^{9}\left((\boldsymbol{u} \times \boldsymbol{v}) \boldsymbol{e}_{i}, \boldsymbol{e}_{i}\right)$, we do straightforward computation of $\operatorname{tr}(\boldsymbol{u} \times \boldsymbol{v})$ :

$$
\begin{aligned}
& \operatorname{tr}(\boldsymbol{u} \times \boldsymbol{v})=\sum_{i=1}^{9}\left((\boldsymbol{u} \times \boldsymbol{v}) \boldsymbol{e}_{i}, \boldsymbol{e}_{i}\right)=\sum_{i=1}^{9}\left(*\left(\boldsymbol{v} \wedge *\left(\boldsymbol{u} \wedge \boldsymbol{e}_{i}\right)\right)+\frac{2}{3}(\boldsymbol{u}, \boldsymbol{v}) \boldsymbol{e}_{i}, \boldsymbol{e}_{i}\right) \\
& =\sum_{i=1}^{9}\left(\left(*\left(v \wedge *\left(u \wedge \boldsymbol{e}_{i}\right)\right), \boldsymbol{e}_{i}\right)+\frac{2}{3}(\boldsymbol{u}, \boldsymbol{v})\left(\boldsymbol{e}_{i}, \boldsymbol{e}_{i}\right)\right) \\
& =\sum_{i=1}^{9}\left(\left(*\left(v \wedge *\left(u \wedge \boldsymbol{e}_{i}\right)\right), \boldsymbol{e}_{i}\right)+6(\boldsymbol{u}, \boldsymbol{v})\right. \\
& =-\sum_{i=1}^{9}\left(e_{i} \wedge u, e_{i} \wedge v\right)+6(u, v)\left(\begin{array}{l}
u=u_{1} \wedge u_{2} \wedge u_{3} \\
v=v_{1} \wedge v_{2} \wedge v_{3}
\end{array}\right) \\
& =-\sum_{i=1}^{9} \operatorname{det}\left(\begin{array}{llll}
\left(\boldsymbol{e}_{i}, \boldsymbol{e}_{i}\right) & \left(\boldsymbol{e}_{i}, \boldsymbol{v}_{1}\right) & \left(\boldsymbol{e}_{i}, \boldsymbol{v}_{2}\right) & \left(\boldsymbol{e}_{i}, \boldsymbol{v}_{3}\right) \\
\left(\boldsymbol{u}_{1}, \boldsymbol{e}_{i}\right) & \left(\boldsymbol{u}_{1}, \boldsymbol{v}_{1}\right) & \left(\boldsymbol{u}_{1}, \boldsymbol{v}_{2}\right) & \left(\boldsymbol{u}_{1}, \boldsymbol{v}_{3}\right) \\
\left(\boldsymbol{u}_{2}, \boldsymbol{e}_{i}\right) & \left(\boldsymbol{u}_{2}, \boldsymbol{v}_{1}\right) & \left(\boldsymbol{u}_{2}, \boldsymbol{v}_{2}\right) & \left(\boldsymbol{u}_{2}, \boldsymbol{v}_{3}\right) \\
\left(\boldsymbol{u}_{3}, \boldsymbol{e}_{i}\right) & \left(\boldsymbol{u}_{3}, \boldsymbol{v}_{1}\right) & \left(\boldsymbol{u}_{3}, \boldsymbol{v}_{2}\right) & \left(\boldsymbol{u}_{3}, \boldsymbol{v}_{3}\right)
\end{array}\right)+6(\boldsymbol{u}, \boldsymbol{v}) \\
& =-\sum_{i=1}^{9}\left(\left(e_{1}, e_{i}\right)(u, v)-\left(e_{i}, v_{1}\right)\left(e_{i} \wedge v_{2} \wedge v_{3}\right)+\left(e_{i}, v_{2}\right)\left(e_{i} \wedge v_{1} \wedge v_{3}\right)\right. \\
& \left.-\left(e_{i}, v_{3}\right)\left(e_{i} \wedge v_{1} \wedge v_{2}\right)\right)+6(u, v) \\
& =-\left(9(u, v)-\left(u, v_{1} \wedge v_{2} \wedge v_{3}\right)+\left(u, v_{2} \wedge v_{1} \wedge v_{3}\right)-\left(u, v_{3} \wedge v_{1} \wedge v_{2}\right)\right) \\
& +6(u, v) \\
& =-\left(9(u, v)-\left(u, v_{1} \wedge v_{2} \wedge v_{3}\right)-\left(u, v_{1} \wedge v_{2} \wedge v_{3}\right)-\left(u, v_{1} \wedge v_{2} \wedge v_{3}\right)\right) \\
& +6(u, v) \\
& =-(9(u, v)-(u, v)-(u, v)-(u, v))+6(u, v) \\
& =0 \text {. }
\end{aligned}
$$


Note that from Lemma 4.2, $\boldsymbol{u} \times \boldsymbol{v}$ can be regarded as an element of $\mathfrak{s l}(9, C)$ with respect to the canonical basis of $C^{9}$.

Lemma 4.3 ([2, Lemma 1.2]). For $A \in S L(9, C), D \in \mathfrak{s l}(9, C)$ and $\boldsymbol{u}, \boldsymbol{v} \in \Lambda^{3}\left(C^{9}\right)$, we have the following relational formulas

(1) $A(\boldsymbol{u} \times \boldsymbol{v}) A^{-1}=A \boldsymbol{u} \times{ }^{t} A^{-1} \boldsymbol{v},[D, \boldsymbol{u} \times \boldsymbol{v}]=D \boldsymbol{u} \times \boldsymbol{v}+\boldsymbol{u} \times\left(-{ }^{t} D \boldsymbol{v}\right)$,

(2) ${ }^{t}(\boldsymbol{u} \times \boldsymbol{v})=\boldsymbol{v} \times \boldsymbol{u}$,

(3) $\tau(\boldsymbol{u} \times \boldsymbol{v})=\tau \boldsymbol{u} \times \tau \boldsymbol{v}$, where $\tau$ is the complex conjugation on $\Lambda^{3}\left(C^{9}\right)$,

(4) $\operatorname{tr}(D(\boldsymbol{u} \times \boldsymbol{v}))=(D \boldsymbol{u}, \boldsymbol{v})$.

Proof. (1) As for the left hand side, it follows from the definition of $\boldsymbol{u} \times \boldsymbol{v}$ that

$$
\begin{aligned}
A(\boldsymbol{u} \times \boldsymbol{v}) A^{-1} \boldsymbol{x} & =A\left(*\left(\boldsymbol{v} \wedge *\left(\boldsymbol{u} \wedge A^{-1} \boldsymbol{x}\right)\right)+\frac{2}{3}(\boldsymbol{u}, \boldsymbol{v})\left(A^{-1} \boldsymbol{x}\right)\right), \boldsymbol{x} \in C^{9} \\
& =A\left(*\left(\boldsymbol{v} \wedge *\left(\boldsymbol{u} \wedge A^{-1} \boldsymbol{x}\right)\right)+\frac{2}{3}(\boldsymbol{u}, \boldsymbol{v}) \boldsymbol{x},\right.
\end{aligned}
$$

on the other hand, as for the right hand side, it follows from Lemma 4.1 that

$$
\begin{aligned}
\left(A \boldsymbol{u} \times{ }^{t} A^{-1}\right) \boldsymbol{x} & =*\left({ }^{t} A^{-1} \boldsymbol{v} \wedge *(A \boldsymbol{u} \wedge \boldsymbol{x})\right)+\frac{2}{3}\left(A \boldsymbol{u},{ }^{t} A^{-1} \boldsymbol{v}\right) \boldsymbol{x}, \boldsymbol{x} \in C^{9} \\
& =*\left({ }^{t} A^{-1} \boldsymbol{v} \wedge *\left(A\left(\boldsymbol{u} \wedge A^{-1} \boldsymbol{x}\right)\right)\right)+\frac{2}{3}(\boldsymbol{u}, \boldsymbol{v}) \boldsymbol{x} \\
& =*\left({ }^{t} A^{-1} \boldsymbol{v} \wedge{ }^{t} A^{-1}\left(*\left(\boldsymbol{u} \wedge A^{-1} \boldsymbol{x}\right)\right)\right)+\frac{2}{3}(\boldsymbol{u}, \boldsymbol{v}) \boldsymbol{x} \\
& =*\left({ }^{t} A^{-1}\left(\boldsymbol{v} \wedge *\left(\boldsymbol{u} \wedge A^{-1} \boldsymbol{x}\right)\right)\right)+\frac{2}{3}(\boldsymbol{u}, \boldsymbol{v}) \boldsymbol{x} \\
& =A\left(*\left(\boldsymbol{v} \wedge *\left(\boldsymbol{u} \wedge A^{-1} \boldsymbol{x}\right)\right)\right)+\frac{2}{3}(\boldsymbol{u}, \boldsymbol{v}) \boldsymbol{x} .
\end{aligned}
$$

Hence we have that $A(\boldsymbol{u} \times \boldsymbol{v}) A^{-1} \boldsymbol{x}=\left(A \boldsymbol{u} \times{ }^{t} A^{-1}\right) \boldsymbol{x}, \boldsymbol{x} \in C^{9}$, that is, $A(\boldsymbol{u} \times \boldsymbol{v}) A^{-1}=$ $A \boldsymbol{u} \times{ }^{t} A^{-1} \boldsymbol{v}$. The relational formula $[D, \boldsymbol{u} \times \boldsymbol{v}]=D \boldsymbol{u} \times \boldsymbol{v}+\boldsymbol{u} \times\left(-{ }^{t} D \boldsymbol{v}\right)$ is shown as above.

(2) Since $\boldsymbol{u} \times \boldsymbol{v} \in \mathfrak{s l}(9, C)($ Lemma 4.2$)$, we have $\left({ }^{t}(\boldsymbol{u} \times \boldsymbol{v}) \boldsymbol{x}, \boldsymbol{y}\right)=(\boldsymbol{x},(\boldsymbol{u} \times \boldsymbol{v}) \boldsymbol{y}), \boldsymbol{x}, \boldsymbol{y} \in$ $C^{9}$. Subsequently, we have to show $(\boldsymbol{x},(\boldsymbol{u} \times \boldsymbol{v}) \boldsymbol{y})=((\boldsymbol{v} \times \boldsymbol{u}) \boldsymbol{x}, \boldsymbol{y})$. Indeed, as for the left hand side, it follows that

$$
\begin{aligned}
(\boldsymbol{x},(\boldsymbol{u} \times \boldsymbol{v}) \boldsymbol{y}) & =\left(\boldsymbol{x}, *(v \wedge *(u \wedge y))+\frac{2}{3}(u, v) y\right) \\
& =(x, *(v \wedge *(u \wedge y)))+\frac{2}{3}(u, v)(x, y) \\
& =(*(v \wedge *(u \wedge y)), x)+\frac{2}{3}(u, v)(x, y) \\
& =-(y \wedge u, x \wedge v)+\frac{2}{3}(u, v)(x, y),
\end{aligned}
$$


on the other hand, as for the right hand side, it follows that

$$
\begin{aligned}
((v \times u) x, y) & =\left(*(u \wedge *(v \wedge x))+\frac{2}{3}(v, u) x, y\right) \\
& =(*(u \wedge *(v \wedge x)), y)+\frac{2}{3}(v, u)(x, y) \\
& =-(x \wedge v, y \wedge u)+\frac{2}{3}(v, u)(x, y) \\
& =-(y \wedge u, x \wedge v)+\frac{2}{3}(u, v)(x, y)
\end{aligned}
$$

Hence we have $\left({ }^{t}(\boldsymbol{u} \times \boldsymbol{v}) \boldsymbol{x}, \boldsymbol{y}\right)=((\boldsymbol{v} \times \boldsymbol{u}) \boldsymbol{x}, \boldsymbol{y})$ for every $\boldsymbol{x}, \boldsymbol{y} \in C^{9}$, that is, ${ }^{t}(\boldsymbol{u} \times \boldsymbol{v})=\boldsymbol{v} \times \boldsymbol{u}$.

(3) Note first that $*(\tau \boldsymbol{u})=\tau(* \boldsymbol{u})$. Indeed, for every $\boldsymbol{v} \in \Lambda^{3}\left(C^{9}\right)$, we have

$$
\begin{aligned}
(*(\tau \boldsymbol{u}), \boldsymbol{v}) & =\left(\tau \boldsymbol{u} \wedge \boldsymbol{v}, \boldsymbol{e}_{1} \wedge \cdots \wedge \boldsymbol{e}_{9}\right) \\
& =\left(\tau(\boldsymbol{u} \wedge \tau \boldsymbol{v}), \tau\left(\boldsymbol{e}_{1} \wedge \cdots \wedge \boldsymbol{e}_{9}\right)\right) \\
& =\tau\left(\boldsymbol{u} \wedge \tau \boldsymbol{v}, \boldsymbol{e}_{1} \wedge \cdots \wedge \boldsymbol{e}_{9}\right) \\
& =\tau(* \boldsymbol{u}, \tau \boldsymbol{v})=(\tau(* \boldsymbol{u}), \tau(\tau \boldsymbol{v})) \\
& =(\tau(* \boldsymbol{u}), \boldsymbol{v}) .
\end{aligned}
$$

Then using this relational formula, it follows that

$$
\begin{aligned}
(\tau \boldsymbol{u} \times \tau \boldsymbol{v}) \boldsymbol{x} & =*(\tau \boldsymbol{v} \wedge *(\tau \boldsymbol{u} \wedge \boldsymbol{x}))+\frac{2}{3}(\tau \boldsymbol{u}, \tau \boldsymbol{v}) \boldsymbol{x}, \boldsymbol{x} \in C^{9} \\
& =*(\tau \boldsymbol{v} \wedge \tau(*(\boldsymbol{u} \wedge \tau \boldsymbol{x})))+\frac{2}{3} \tau(\boldsymbol{u}, \boldsymbol{v}) \boldsymbol{x} \\
& =\tau(*(\boldsymbol{v} \wedge *(\boldsymbol{u} \wedge \tau \boldsymbol{x})))+\frac{2}{3} \tau(\boldsymbol{u}, \boldsymbol{v}) \boldsymbol{x} \\
& =\tau\left(*(\boldsymbol{v} \wedge *(\boldsymbol{u} \wedge \tau \boldsymbol{x}))+\frac{2}{3}(\boldsymbol{u}, \boldsymbol{v})(\tau \boldsymbol{x})\right) \\
& =\tau((\boldsymbol{u} \times \boldsymbol{v})(\tau \boldsymbol{x})) \\
& =\tau(\boldsymbol{u} \times \boldsymbol{v}) \boldsymbol{x}
\end{aligned}
$$

for every $\boldsymbol{x} \in C^{9}$. Hence we have $\tau(\boldsymbol{u} \times \boldsymbol{v})=\tau \boldsymbol{u} \times \tau \boldsymbol{v}$.

(4) First, as in (1) above, we do straightforward computation of $D(\boldsymbol{u} \times \boldsymbol{v}) \boldsymbol{x}, \boldsymbol{x} \in C^{9}$. It follows from Lemma 4.1 that

$$
\begin{aligned}
D(\boldsymbol{u} \times \boldsymbol{v}) \boldsymbol{x} & =D\left(*(\boldsymbol{v} \wedge *(\boldsymbol{u} \wedge \boldsymbol{x}))+\frac{2}{3}(\boldsymbol{u}, \boldsymbol{v}) \boldsymbol{x}\right) \\
& =D(*(\boldsymbol{v} \wedge *(\boldsymbol{u} \wedge \boldsymbol{x})))+\frac{2}{3}(\boldsymbol{u}, \boldsymbol{v})(D \boldsymbol{x}) \\
& =*\left(-{ }^{t} D(\boldsymbol{v} \wedge *(\boldsymbol{u} \wedge \boldsymbol{x}))\right)+\frac{2}{3}(\boldsymbol{u}, \boldsymbol{v})(D \boldsymbol{x}) \\
& =*\left(-{ }^{t} D \boldsymbol{v} \wedge *(\boldsymbol{u} \wedge \boldsymbol{x})+\boldsymbol{v} \wedge *(D \boldsymbol{u} \wedge \boldsymbol{x})+\boldsymbol{v} \wedge *(\boldsymbol{u} \wedge D \boldsymbol{x})\right)+\frac{2}{3}(\boldsymbol{u}, \boldsymbol{v}) D \boldsymbol{x}
\end{aligned}
$$


Then, as in the proof of Lemma 4.2, note that $\operatorname{tr}(D)=0$, we have the following

$$
\begin{aligned}
\operatorname{tr}(D(\boldsymbol{u} \times \boldsymbol{v}))= & \sum_{j=1}^{9}\left(D(\boldsymbol{u} \times \boldsymbol{v}) \boldsymbol{e}_{j}, \boldsymbol{e}_{j}\right) \\
= & \sum_{j=1}^{9}\left(*\left(-{ }^{t} D \boldsymbol{v} \wedge *\left(\boldsymbol{u} \wedge \boldsymbol{e}_{j}\right)+\boldsymbol{v} \wedge *\left(D \boldsymbol{u} \wedge \boldsymbol{e}_{j}\right)+\boldsymbol{v} \wedge *\left(\boldsymbol{u} \wedge D \boldsymbol{e}_{j}\right)\right)\right. \\
& \left.+\frac{2}{3}(\boldsymbol{u}, \boldsymbol{v}) D \boldsymbol{e}_{j}, \boldsymbol{e}_{j}\right) \\
= & \sum_{j=1}^{9}\left(*\left(-{ }^{t} D \boldsymbol{v} \wedge *\left(\boldsymbol{u} \wedge \boldsymbol{e}_{j}\right)+\boldsymbol{v} \wedge *\left(D \boldsymbol{u} \wedge \boldsymbol{e}_{j}\right)+\boldsymbol{v} \wedge *\left(\boldsymbol{u} \wedge D \boldsymbol{e}_{j}\right)\right), \boldsymbol{e}_{j}\right) \\
& +\frac{2}{3}(\boldsymbol{u}, \boldsymbol{v}) \sum_{j=1}^{9}\left(D \boldsymbol{e}_{j}, \boldsymbol{e}_{j}\right) \\
= & \sum_{j=1}^{9}\left(*\left(-{ }^{t} D \boldsymbol{v} \wedge *\left(\boldsymbol{u} \wedge \boldsymbol{e}_{j}\right)+\boldsymbol{v} \wedge *\left(D \boldsymbol{u} \wedge \boldsymbol{e}_{j}\right)+\boldsymbol{v} \wedge *\left(\boldsymbol{u} \wedge D \boldsymbol{e}_{j}\right)\right), \boldsymbol{e}_{j}\right) \\
& +\frac{2}{3}(\boldsymbol{u}, \boldsymbol{v}) \operatorname{tr}(D) \\
= & \sum_{j=1}^{9}\left(*\left(-{ }^{t} D \boldsymbol{v} \wedge *\left(\boldsymbol{u} \wedge \boldsymbol{e}_{j}\right)+\boldsymbol{v} \wedge *\left(D \boldsymbol{u} \wedge \boldsymbol{e}_{j}\right)+\boldsymbol{v} \wedge *\left(\boldsymbol{u} \wedge D \boldsymbol{e}_{j}\right)\right), \boldsymbol{e}_{j}\right) \\
= & \sum_{j=1}^{9}\left(\left(*\left(-{ }^{t} D \boldsymbol{v} \wedge *\left(\boldsymbol{u} \wedge \boldsymbol{e}_{j}\right)\right), \boldsymbol{e}_{j}\right)+\left(*\left(\boldsymbol{v} \wedge *\left(D \boldsymbol{u} \wedge \boldsymbol{e}_{j}\right)\right), \boldsymbol{e}_{j}\right)\right. \\
= & \left.\left.+\sum_{j=1}^{9}\left(\left(\boldsymbol{e}_{j} \wedge \boldsymbol{u}, \boldsymbol{e}_{j} \wedge-{ }^{t} D \boldsymbol{v}\right)+\left(\boldsymbol{e}_{j} \wedge D \boldsymbol{u}, \boldsymbol{e}_{j} \wedge \boldsymbol{v}\right)+\left(D \wedge D \boldsymbol{e}_{j}\right)\right), \boldsymbol{e}_{j}\right)\right) \\
&
\end{aligned}
$$

Here, as for computation above, we do the following computation:

$$
\begin{aligned}
\left(\boldsymbol{e}_{j} \wedge \boldsymbol{u}, \boldsymbol{e}_{j} \wedge-{ }^{t} D \boldsymbol{v}\right) & =\left(\boldsymbol{e}_{j} \wedge \boldsymbol{u},-{ }^{t} D\left(\boldsymbol{e}_{j} \wedge \boldsymbol{v}\right)+{ }^{t} D \boldsymbol{e}_{j} \wedge \boldsymbol{v}\right) \\
& =\left(\boldsymbol{e}_{j} \wedge \boldsymbol{u},-{ }^{t} D\left(\boldsymbol{e}_{j} \wedge \boldsymbol{v}\right)\right)+\left(\boldsymbol{e}_{j} \wedge \boldsymbol{u},{ }^{t} D \boldsymbol{e}_{j} \wedge \boldsymbol{v}\right) \\
& =-\left(D\left(\boldsymbol{e}_{j} \wedge \boldsymbol{u}\right), \boldsymbol{e}_{j} \wedge \boldsymbol{v}\right)+\left(\boldsymbol{e}_{j} \wedge \boldsymbol{u},{ }^{t} D \boldsymbol{e}_{j} \wedge \boldsymbol{v}\right) \\
& =-\left(D \boldsymbol{e}_{j} \wedge \boldsymbol{u}+\boldsymbol{e}_{j} \wedge D \boldsymbol{u}, \boldsymbol{e}_{j} \wedge \boldsymbol{v}\right)+\left(\boldsymbol{e}_{j} \wedge \boldsymbol{u},{ }^{t} D \boldsymbol{e}_{j} \wedge \boldsymbol{v}\right) \\
& =-\left(D \boldsymbol{e}_{j} \wedge \boldsymbol{u}, \boldsymbol{e}_{j} \wedge \boldsymbol{v}\right)-\left(\boldsymbol{e}_{j} \wedge D \boldsymbol{u}, \boldsymbol{e}_{j} \wedge \boldsymbol{v}\right)+\left(\boldsymbol{e}_{j} \wedge \boldsymbol{u},{ }^{t} D \boldsymbol{e}_{j} \wedge \boldsymbol{v}\right)
\end{aligned}
$$

Hence, combining both computations above, we have

$$
\operatorname{tr}(D(\boldsymbol{u} \times \boldsymbol{v}))=-\sum_{j=1}^{9}\left(\boldsymbol{e}_{j} \wedge \boldsymbol{u},{ }^{t} D \boldsymbol{e}_{j} \wedge \boldsymbol{v}\right) .
$$

Thus, as in the proof of Lemma 4.2, we obtain $\operatorname{tr}(D(\boldsymbol{u} \times \boldsymbol{v}))=(D \boldsymbol{u}, \boldsymbol{v})$. 
Note that Theorems 4.1, 4.2 and 4.3 also hold with respect to an exterior $C$-vector space $\Lambda^{k}\left(C^{n}\right)$ and an $n$-dimensional $C$-vector space $C^{n}$, and accordingly, the Lie algebra $\mathfrak{s l}(9, C)$ and the group $S L(9, C)$ are replaced by the Lie algebra $\mathfrak{s l}(n, C)$ and the group $S L(n, C)$ (see [2] for details).

Now, we construct another $C$-Lie algebra $e_{8}{ }^{C}$ of type $E_{8}$. Hereafter, we use the same notation $\mathrm{e}_{8}{ }^{C}$ used in previous section as $C$-Lie algebra of type $E_{8}$.

Theorem 4.4 ([2, pp.599-600]). In the 248-dimensional C-vector space

$$
\mathrm{e}_{8}{ }^{C}=\mathfrak{s l}(9, C) \oplus \Lambda^{3}\left(C^{9}\right) \oplus \Lambda^{3}\left(C^{9}\right),
$$

for $R_{1}=\left(D_{1}, \boldsymbol{u}_{1}, \boldsymbol{v}_{1}\right), R_{2}=\left(D_{2}, \boldsymbol{u}_{2}, \boldsymbol{v}_{2}\right) \in \mathrm{e}_{8}{ }^{C}$, we define a Lie bracket $\left[R_{1}, R_{2}\right]$ by

$$
\left[\left(D_{1}, \boldsymbol{u}_{1}, \boldsymbol{v}_{1}\right),\left(D_{2}, \boldsymbol{u}_{2}, \boldsymbol{v}_{2}\right)\right]=:(D, \boldsymbol{u}, \boldsymbol{v}),
$$

where

$$
\left\{\begin{array}{l}
D=\left[D_{1}, D_{2}\right]+\boldsymbol{u}_{1} \times \boldsymbol{v}_{2}-\boldsymbol{u}_{2} \times \boldsymbol{v}_{1} \\
\boldsymbol{u}=D_{1} \boldsymbol{u}_{2}-D_{2} \boldsymbol{u}_{1}+*\left(\boldsymbol{v}_{1} \wedge \boldsymbol{v}_{2}\right) \\
\boldsymbol{v}=-{ }^{t} D_{1} \boldsymbol{v}_{2}+{ }^{t} D_{2} \boldsymbol{v}_{1}-*\left(\boldsymbol{u}_{1} \wedge \boldsymbol{u}_{2}\right)
\end{array}\right.
$$

then $\mathrm{e}_{8}{ }^{C}$ becomes a $C$-Lie algebra.

In order to prove the Jacobi identity, we need the following Lemma.

Lemma 4.5 ([2, Lemma 2.1]). For $\boldsymbol{u}, \boldsymbol{v}, \boldsymbol{w} \in \Lambda^{3}\left(C^{9}\right)$, we have the following

(1) $\boldsymbol{u} \times *(\boldsymbol{v} \wedge \boldsymbol{w})+\boldsymbol{v} \times *(\boldsymbol{w} \wedge \boldsymbol{u})+\boldsymbol{w} \times *(\boldsymbol{u} \wedge \boldsymbol{v})=0$.

(2) $(\boldsymbol{u} \times \boldsymbol{w}) \boldsymbol{v}-(\boldsymbol{v} \times \boldsymbol{w}) \boldsymbol{u}+*(*(\boldsymbol{u} \wedge \boldsymbol{v}) \wedge \boldsymbol{w})=0$.

We will return the proof of Theorem 4.4 .

Proof. We start the proof of theorem. Let $R_{i}=\left(D_{i}, \boldsymbol{u}_{i}, \boldsymbol{v}_{i}\right), i=1,2$ and $R_{3} \in \mathrm{e}_{8}{ }^{C}$. First, it is clear that $\left[R_{1}+R_{2}, R_{3}\right]=\left[R_{1}, R_{3}\right]+\left[R_{2}, R_{3}\right]$ and $\left[\lambda R_{1}, R_{2}\right]=\lambda\left[R_{1}, R_{2}\right], \lambda \in C$. Next, for $\boldsymbol{u}, \boldsymbol{v} \in \Lambda^{3}\left(C^{9}\right)$, since we confirm $*(\boldsymbol{u} \times \boldsymbol{v})=-*(\boldsymbol{v} \times \boldsymbol{u})$, we have $\left[R_{1}, R_{2}\right]=-\left[R_{2}, R_{1}\right]$. Finally, we have to prove the Jacob identity. In order to prove this, using Lemmas 4.1. 4.3, 4.5 and note that the relational formulas $*\left(\boldsymbol{v}_{i} \wedge *\left(\boldsymbol{u}_{k} \wedge \boldsymbol{u}_{l}\right)\right)=-*\left(*\left(\boldsymbol{u}_{k} \wedge \boldsymbol{u}_{l}\right) \wedge\right.$ $\left.\boldsymbol{v}_{k}\right), D_{i} *\left(\boldsymbol{v}_{k} \wedge \boldsymbol{v}_{l}\right)+*\left(\boldsymbol{v}_{l} \wedge\left(-{ }^{t} D_{i} \boldsymbol{v}_{k}\right)\right)+*\left(\boldsymbol{v}_{k} \wedge{ }^{t} D_{i} \boldsymbol{v}_{l}\right)=0$ hold, it follows from

$$
\begin{aligned}
& {\left[R_{i},\left[R_{k}, R_{l}\right]\right]=:(D, \boldsymbol{u}, \boldsymbol{v}),} \\
& D=\left[D_{i},\left[D_{k}, D_{l}\right]\right]+D_{i} \boldsymbol{u}_{k} \times \boldsymbol{v}_{l}+\boldsymbol{u}_{1} \times\left({ }^{t} D_{i} \boldsymbol{v}_{l}\right)-D_{i} \boldsymbol{u}_{l} \times \boldsymbol{v}_{k}-\boldsymbol{u}_{l} \times\left({ }^{t} D_{i} \boldsymbol{v}_{k}\right) \\
& -\boldsymbol{u}_{i} \times{ }^{t} D_{k} \boldsymbol{v}_{l}+\boldsymbol{u}_{i} \times{ }^{t} D_{l} \boldsymbol{v}_{k}-\boldsymbol{u}_{i} \times\left(*\left(\boldsymbol{u}_{k} \wedge \boldsymbol{u}_{l}\right)\right) \\
& -D_{k} \boldsymbol{u}_{l} \times \boldsymbol{v}_{i}-\left(*\left(\boldsymbol{v}_{k} \wedge \boldsymbol{v}_{l}\right)\right) \times \boldsymbol{v}_{i}, \\
& \boldsymbol{u}=D_{i} D_{k} \boldsymbol{u}_{l}-D_{i} D_{l} \boldsymbol{u}_{k}+D_{i} *\left(\boldsymbol{v}_{k} \wedge \boldsymbol{v}_{l}\right)-\left[D_{k}, D_{l}\right] \boldsymbol{u}_{i}-\left(\boldsymbol{u}_{k} \times \boldsymbol{v}_{l}\right) \boldsymbol{u}_{i} \\
& +\left(\boldsymbol{u}_{l} \times \boldsymbol{v}_{k}\right) \boldsymbol{u}_{i}+*\left(\boldsymbol{v}_{i} \wedge\left({ }^{t} D_{k} \boldsymbol{v}_{l}\right)\right)+*\left(\boldsymbol{v}_{i} \wedge{ }^{t} D_{l} \boldsymbol{v}_{k}\right)-*\left(\boldsymbol{v}_{i} \wedge\left(\boldsymbol{u}_{k} \wedge \boldsymbol{u}_{l}\right)\right), \\
& \boldsymbol{v}={ }^{t} D_{i}{ }^{t} D_{k} \boldsymbol{v}_{l}-{ }^{t} D_{i}{ }^{t} D_{l} \boldsymbol{v}_{1}+{ }^{t} D_{i} *\left(\boldsymbol{u}_{i} \wedge \boldsymbol{u}_{l}\right)+{ }^{t}\left[D_{k}, D_{l}\right] \boldsymbol{v}_{i}+{ }^{t}\left(\boldsymbol{u}_{k} \times \boldsymbol{v}_{l}\right) \boldsymbol{v}_{i} \\
& -{ }^{t}\left(\boldsymbol{u}_{l} \times \boldsymbol{v}_{k}\right) \boldsymbol{v}_{i}-*\left(\boldsymbol{u}_{i} \wedge D_{k} \boldsymbol{u}_{l}\right)+*\left(\boldsymbol{u}_{i} \wedge D_{l} \boldsymbol{u}_{k}\right)-*\left(\boldsymbol{u}_{i} \wedge *\left(\boldsymbol{v}_{k} \wedge \boldsymbol{v}_{l}\right)\right)
\end{aligned}
$$

that the Jacob identity $\left[R_{1},\left[R_{2}, R_{3}\right]\right]+\left[R_{2},\left[R_{3}, R_{1}\right]\right]+\left[R_{3},\left[R_{1}, R_{2}\right]\right]=0$. 
Here, we need the following results.

Theorem 4.6 ([2, Theorem 2.2]). The C-Lie algebra $\mathrm{e}_{8}{ }^{C}=\mathfrak{s l}(9, C) \oplus \Lambda^{3}\left(C^{9}\right) \oplus \Lambda^{3}\left(C^{9}\right)$ is a simple Lie algebra of type $E_{8}$.

Proposition 4.7 ([2, p.602]). The Killing form $B_{8}$ of the C-Lie algebra $\mathrm{e}_{8}{ }^{C}=\mathfrak{s l}(9, C) \oplus$ $\Lambda^{3}\left(C^{9}\right) \oplus \Lambda^{3}\left(C^{9}\right)$ is given by

$$
B_{8}\left(\left(D_{1}, \boldsymbol{u}_{1}, \boldsymbol{v}_{1}\right),\left(D_{2}, \boldsymbol{u}_{2}, \boldsymbol{v}_{2}\right)\right)=60\left(\operatorname{tr}\left(D_{1} D_{2}\right)+\left(\boldsymbol{u}_{1}, \boldsymbol{v}_{2}\right)+\left(\boldsymbol{u}_{2}, \boldsymbol{v}_{1}\right)\right)
$$

We define a complex-conjugate linear transformation $\tau \tilde{\lambda}$ of $e_{8}{ }^{C}$ by

$$
\tau \tilde{\lambda}(D, \boldsymbol{u}, \boldsymbol{v})=\left(-\tau^{t} D,-\tau \boldsymbol{v},-\tau \boldsymbol{u}\right),
$$

and using this linear transformation we define an Hermitian inner product $\left\langle R_{1}, R_{2}\right\rangle$ in $\mathrm{e}_{8}{ }^{C}$ by

$$
\left\langle R_{1}, R_{2}\right\rangle=-B_{8}\left(R_{1}, \tau \tilde{\lambda} R_{2}\right) .
$$

Then the explicit form of $\left\langle R_{1}, R_{2}\right\rangle$ is given as follows:

$$
\left\langle R_{1}, R_{2}\right\rangle=60\left(\operatorname{tr}\left(D_{1}\left(\tau^{t} D_{2}\right)\right)+\left(\boldsymbol{u}_{1}, \tau \boldsymbol{u}_{2}\right)+\left(\boldsymbol{v}_{2}, \tau \boldsymbol{v}_{1}\right)\right) .
$$

Consequently, as mentioned in [2, p.598], the group

$$
E_{8}=\left\{\alpha \in \operatorname{Aut}\left(\mathrm{e}_{8}{ }^{C}\right) \mid\left\langle\alpha R_{1}, \alpha R_{2}\right\rangle=\left\langle R_{1}, R_{2}\right\rangle\right\}
$$

is the connected compact simple Lie group of type $E_{8}$ and also simply connected.

Then we have the following lemma.

Lemma 4.8. The Lie algebra $\mathrm{e}_{8}$ of the group $E_{8}$ is given by

$$
\begin{aligned}
\mathrm{e}_{8} & =\left\{R \in \mathfrak{e}_{8}{ }^{C} \mid \tau \tilde{\lambda} R=R\right\} \\
& =\left\{R=(D, \boldsymbol{u},-\tau \boldsymbol{u}) \in \mathfrak{e}_{8}{ }^{C} \mid D \in \mathfrak{s u}(9), \boldsymbol{u} \in \Lambda^{3}\left(C^{9}\right)\right\} .
\end{aligned}
$$

Proof. By doing straightforward computation, we can confirm the required result.

We define a $C$-linear transformation $w_{4}$ of $\mathrm{e}_{8}{ }^{C}$ by

$$
w_{4}(D, \boldsymbol{u}, \boldsymbol{v})=\left(A_{4} D A_{4}{ }^{-1}, A_{4} \boldsymbol{u},{ }^{t} A_{4}{ }^{-1} \boldsymbol{v}\right),
$$

where $A_{4}=\operatorname{diag}(1, i, \ldots, i) \in S U(9)=\left\{A \in M(9, C) \mid\left(\tau^{t} A\right) A=E, \operatorname{det} A=1\right\}$. Then we see that $w_{4} \in E_{8}$ and $\left(w_{4}\right)^{4}=1$. Hence $w_{4}$ induces the inner automorphism $\tilde{w}_{4}$ of order four on $E_{8}: \tilde{w}_{4}(\alpha)=w_{4} \alpha w_{4}^{-1}, \alpha \in E_{8}$.

Now, we will study the subgroup $\left(E_{8}\right)^{w_{4}}$ of $E_{8}$ :

$$
\left(E_{8}\right)^{w_{4}}=\left\{\alpha \in E_{8} \mid w_{4} \alpha=\alpha w_{4}\right\} .
$$

The aim of the rest of this section is to determine the structure of the group $\left(E_{8}\right)^{w_{4}}$. Before that, we prove lemma needed later.

Lemma 4.9. The Lie algebra $\left(\mathfrak{e}_{8}\right)^{w_{4}}$ of the group $\left(E_{8}\right)^{w_{4}}$ is given by

$$
\left(\mathfrak{e}_{8}\right)^{w_{4}}=\left\{(D, 0,0) \in \mathfrak{e}_{8} \mid D \in \mathfrak{s}(\mathfrak{u}(1) \oplus \mathfrak{u}(8))\right\} .
$$

In particular, we have $\operatorname{dim}\left(\left(\mathrm{e}_{8}\right)^{w_{4}}\right)=(64+1)-1=64$. 
Proof. By doing straightforward computation, we can obtain the explicit form above of the Lie algebra $\left(e_{8}\right)^{w_{4}}$. Indeed, it follows that

$$
\begin{aligned}
\left(\mathfrak{e}_{8}\right)^{w_{4}} & =\left\{(D, \boldsymbol{u},-\tau \boldsymbol{u}) \in \mathfrak{e}_{8} \mid w_{4}(D, \boldsymbol{u},-\tau \boldsymbol{u})=(D, \boldsymbol{u},-\tau \boldsymbol{u})\right\} \\
& =\left\{(D, \boldsymbol{u},-\tau \boldsymbol{u}) \in \mathfrak{e}_{8} \mid A_{4} D=D A_{4}, A_{4} \boldsymbol{u}=\boldsymbol{u},{ }^{t} A_{4}{ }^{-1}(-\tau \boldsymbol{u})=-\tau \boldsymbol{u}\right\} \\
& =\left\{(D, \boldsymbol{u},-\tau \boldsymbol{u}) \in \mathfrak{e}_{8} \mid A_{4} D=D A_{4}, A_{4} \boldsymbol{u}=\boldsymbol{u}\right\} \\
& =\left\{(D, 0,0) \in \mathfrak{e}_{8} \mid D \in \mathfrak{s}(\mathfrak{u}(1) \oplus \mathfrak{u}(8))\right\} .
\end{aligned}
$$

It is clear that $\operatorname{dim}\left(\left(\mathrm{e}_{8}\right)^{w_{4}}\right)=(64+1)-1=64$.

Now, we will determine the structure of the group $\left(E_{8}\right)^{w_{4}}$.

Theorem 4.10. The group $\left(E_{8}\right)^{w_{4}}$ is isomorphic to the group $(U(1) \times S U(8)) / \boldsymbol{Z}_{24}, \boldsymbol{Z}_{24}$ $=\left\{\left(\omega_{24}{ }^{3 k}, \omega_{24}{ }^{-3 k} E\right),\left(\omega_{24}{ }^{3 k+1}, \omega_{24}{ }^{-3(k-5)} E\right),\left(\omega_{24}{ }^{3 k+2}, \omega_{24}{ }^{-3(k-2)} E\right) \mid k=0, \ldots, 7\right\}, \omega_{24}$ $=e^{i 2 \pi / 24}:\left(E_{8}\right)^{w_{4}} \cong(U(1) \times S U(8)) / Z_{24}$.

Proof. We define a mapping $\varphi_{w_{4}}: S(U(1) \times U(8)) \rightarrow\left(E_{8}\right)^{w_{4}}$ by

$$
\varphi_{w_{4}}(A)(D, \boldsymbol{u}, \boldsymbol{v})=\left(A D A^{-1}, A \boldsymbol{u},{ }^{t} A^{-1} \boldsymbol{v}\right) .
$$

We will prove that $\varphi_{w_{4}}$ is well-defined. For $R_{1}=\left(D_{1}, \boldsymbol{u}_{1}, \boldsymbol{v}_{1}\right), R_{2}=\left(D_{2}, \boldsymbol{u}_{2}, \boldsymbol{v}_{2}\right) \in \mathrm{e}_{8}{ }^{C}$, we first show $\varphi_{w_{4}}(A)\left[R_{1}, R_{2}\right]=\left[\varphi_{w_{4}}(A)\left(R_{1}\right), \varphi_{w_{4}}(A)\left(R_{2}\right)\right]$. It follows from Lemmas 4.1 (2), 4.3 (1) that

$$
\varphi_{w_{4}}(A)\left[R_{1}, R_{2}\right]=\varphi_{w_{4}}(A)\left(\left[\left(D_{1}, \boldsymbol{u}_{1}, \boldsymbol{v}_{1}\right),\left(D_{2}, \boldsymbol{u}_{2}, \boldsymbol{v}_{2}\right)\right]\right)=:\left(A D A^{-1}, A \boldsymbol{u},{ }^{t} A^{-1} \boldsymbol{v}\right),
$$

where

$$
\begin{aligned}
A D A^{-1} & =A\left(\left[D_{1}, D_{2}\right]+\boldsymbol{u}_{1} \times \boldsymbol{v}_{2}-\boldsymbol{u}_{2} \times \boldsymbol{v}_{1}\right) A^{-1} \\
& \left.\left.\left.=A\left[D_{1}, D_{2}\right]\right) A^{-1}+A\left(\boldsymbol{u}_{1} \times \boldsymbol{v}_{2}\right)\right) A^{-1}-A\left(\boldsymbol{u}_{2} \times \boldsymbol{v}_{1}\right)\right) A^{-1} \\
& =A\left(D_{1} D_{2}-D_{2} D_{1}\right) A^{-1}+A \boldsymbol{u}_{1} \times{ }^{t} A^{-1} \boldsymbol{v}_{2}-A \boldsymbol{u}_{2} \times{ }^{t} A^{-1} \boldsymbol{v}_{1} \\
& =\left[A D_{1} A^{-1}, A D_{2} A^{-1}\right]+A \boldsymbol{u}_{1} \times{ }^{t} A^{-1} \boldsymbol{v}_{2}-A \boldsymbol{u}_{2} \times{ }^{t} A^{-1} \boldsymbol{v}_{1}, \\
A \boldsymbol{u} & =A\left(D_{1} \boldsymbol{u}_{2}-D_{2} \boldsymbol{u}_{1}+*\left(\boldsymbol{v}_{1} \wedge \boldsymbol{v}_{2}\right)\right) \\
& =A D_{1} \boldsymbol{u}_{2}-A D_{2} \boldsymbol{u}_{1}+A\left(*\left(\boldsymbol{v}_{1} \wedge \boldsymbol{v}_{2}\right)\right) \\
& =\left(A D_{1}\right) \boldsymbol{u}_{2}-\left(A D_{2}\right) \boldsymbol{u}_{1}+*\left({ }^{t} A^{-1}\left(\boldsymbol{v}_{1} \wedge \boldsymbol{v}_{2}\right)\right) \\
& =\left(A D_{1}\right) \boldsymbol{u}_{2}-\left(A D_{2}\right) \boldsymbol{u}_{1}+*\left({ }^{t} A^{-1} \boldsymbol{v}_{1} \wedge{ }^{t} A^{-1} \boldsymbol{v}_{2}\right), \\
{ }^{t} A^{-1} \boldsymbol{v} & ={ }^{t} A^{-1}\left(-{ }^{t} D_{1} \boldsymbol{v}_{2}+{ }^{t} D_{2} \boldsymbol{v}_{1}-*\left(\boldsymbol{u}_{1} \wedge \boldsymbol{u}_{2}\right)\right) \\
& ={ }^{t} A^{-1}\left(-{ }^{t} D_{1} \boldsymbol{v}_{2}\right)+{ }^{t} A^{-1 t} D_{2} \boldsymbol{v}_{1}-{ }^{t} A^{-1}\left(*\left(\boldsymbol{u}_{1} \wedge \boldsymbol{u}_{2}\right)\right) \\
& =\left({ }^{t} A^{-1}\left(-{ }^{t} D_{1}\right)\right) \boldsymbol{v}_{2}+\left({ }^{t} A^{-1 t} D_{2}\right) \boldsymbol{v}_{1}-*\left(A \boldsymbol{u}_{1} \wedge A \boldsymbol{u}_{2}\right),
\end{aligned}
$$

and

$$
\left[\varphi_{w_{4}}(A)\left(R_{1}\right), \varphi_{w_{4}}(A)\left(R_{2}\right)\right]=\left[\varphi_{w_{4}}(A)\left(D_{1}, \boldsymbol{u}_{1}, \boldsymbol{v}_{1}\right), \varphi_{w_{4}}(A)\left(D_{2}, \boldsymbol{u}_{2}, \boldsymbol{v}_{2}\right)\right]
$$




$$
\begin{aligned}
& =\left[\left(A D_{1} A^{-1}, A \boldsymbol{u}_{1},{ }^{t} A^{-1} \boldsymbol{v}_{1}\right),\left(A D_{2} A^{-1}, A \boldsymbol{u}_{2},{ }^{t} A^{-1} \boldsymbol{v}_{2}\right)\right] \\
& =:\left(D^{\prime}, \boldsymbol{u}^{\prime}, \boldsymbol{v}^{\prime}\right)
\end{aligned}
$$

where

$$
\begin{aligned}
D^{\prime} & =\left[A D_{1} A^{-1}, A D_{2} A^{-1}\right]+A \boldsymbol{u}_{1} \times{ }^{t} A^{-1} \boldsymbol{v}_{2}-A \boldsymbol{u}_{2} \times{ }^{t} A^{-1} \boldsymbol{v}_{1}, \\
\boldsymbol{u}^{\prime} & =\left(A D_{1} A^{-1}\right)\left(A \boldsymbol{u}_{1}\right)-\left(A D_{2} A^{-1}\right)\left(A \boldsymbol{u}_{2}\right)+*\left({ }^{t} A^{-1} \boldsymbol{v}_{1} \wedge{ }^{t} A^{-1} \boldsymbol{v}_{2}\right) \\
& =\left(A D_{1}\right) \boldsymbol{u}_{2}-\left(A D_{2}\right) \boldsymbol{u}_{1}+*\left({ }^{t} A^{-1} \boldsymbol{v}_{1} \wedge{ }^{t} A^{-1} \boldsymbol{v}_{2}\right), \\
\boldsymbol{v}^{\prime} & =-{ }^{t}\left(A D_{1} A^{-1}\right)\left({ }^{t} A^{-1} \boldsymbol{v}_{2}\right)+{ }^{t}\left(A D_{2} A^{-1}\right)\left({ }^{t} A^{-1} \boldsymbol{v}_{1}\right)-*\left(A \boldsymbol{u}_{1} \wedge A \boldsymbol{u}_{2}\right) \\
& =\left({ }^{t} A^{-1}\left(-{ }^{t} D_{1}\right)\right) \boldsymbol{v}_{2}+\left({ }^{t} A^{-1 t} D_{2}\right) \boldsymbol{v}_{1}-*\left(A \boldsymbol{u}_{1} \wedge A \boldsymbol{u}_{2}\right) .
\end{aligned}
$$

Hence we have $\varphi_{w_{4}}(A)\left(\left[R_{1}, R_{2}\right]\right)=\left[\varphi_{w_{4}}(A)\left(R_{1}\right), \varphi_{w_{4}}(A)\left(R_{2}\right)\right]$.

Next, we will show $\left\langle\varphi_{w_{4}}(A)\left(R_{1}\right), \varphi_{w_{4}}(A)\left(R_{2}\right)\right\rangle=\left\langle R_{1}, R_{2}\right\rangle$. It follows that

$$
\begin{aligned}
\left\langle\varphi_{w_{4}}(A)\left(R_{1}\right), \varphi_{w_{4}}(A)\left(R_{2}\right)\right\rangle= & \left\langle\left(A D_{1} A^{-1}, A \boldsymbol{u}_{1},{ }^{t} A^{-1} \boldsymbol{v}_{1}\right),\left(A D_{2} A^{-1}, A \boldsymbol{u}_{2},{ }^{t} A^{-1} \boldsymbol{v}_{2}\right)\right\rangle \\
= & 60\left(\left(\operatorname{tr}\left(A D_{1} A^{-1}\right) \tau^{t}\left(A D_{2} A^{-1}\right)\right)+\left(A \boldsymbol{u}_{1}, \tau\left(A \boldsymbol{u}_{2}\right)\right.\right. \\
& +\left({ }^{t} A^{-1} \boldsymbol{v}_{2}, \tau\left({ }^{t} A^{-1} \boldsymbol{v}_{1}\right)\right) \\
& =60\left(\left(\operatorname{tr}\left(A D_{1} A^{-1}\left(\tau^{t} A^{-1}\right)\left(\tau^{t} D_{2}\right)\left(\tau^{t} A\right)\right)+\left(A \boldsymbol{u}_{1},(\tau A)\left(\tau \boldsymbol{u}_{2}\right)\right)\right.\right. \\
& +\left({ }^{t} A^{-1} \boldsymbol{v}_{2},\left(\tau^{t} A^{-1}\right)\left(\tau v_{1}\right)\right) \\
= & 60\left(\operatorname{tr}\left(\left(A D_{1}\right)\left(\left(\tau^{t} D_{2}\right)\left(\tau^{t} A\right)\right)\right)+\left(\left(\tau^{t} A A\right) \boldsymbol{u}_{1}, \tau \boldsymbol{u}_{2}\right)\right. \\
& \left.+\left(\left(\tau A^{-1 t} A^{-1}\right) \boldsymbol{v}_{2}, \tau \boldsymbol{v}_{1}\right)\right) \\
= & 60\left(\operatorname{tr}\left(\left(\tau^{t} D_{2}\right)\left(\tau^{t} A\right)\right)\left(A D_{1}\right)+\left(\boldsymbol{u}_{1}, \tau \boldsymbol{u}_{2}\right)+\left(\boldsymbol{v}_{2}, \tau \boldsymbol{v}_{1}\right)\right) \\
= & 60\left(\operatorname{tr}\left(\left(\tau^{t} D_{2}\right) D_{1}\right)+\left(\boldsymbol{u}_{1}, \tau \boldsymbol{u}_{2}\right)+\left(\boldsymbol{v}_{2}, \tau \boldsymbol{v}_{1}\right)\right) \\
= & 60\left(\operatorname{tr}\left(D_{1}\left(\tau^{t} D_{2}\right)\right)+\left(\boldsymbol{u}_{1}, \tau \boldsymbol{u}_{2}\right)+\left(\boldsymbol{v}_{2}, \tau \boldsymbol{v}_{1}\right)\right) \\
= & \left\langle R_{1}, R_{2}\right\rangle,
\end{aligned}
$$

that is, $\left\langle\varphi_{w_{4}}(A)\left(R_{1}\right), \varphi_{w_{4}}(A)\left(R_{2}\right)\right\rangle=\left\langle R_{1}, R_{2}\right\rangle$. Hence we have $\varphi_{w_{4}}(A) \in E_{8}$. Moreover, since $A A_{4}=A_{4} A$ holds, we have $w_{4} \varphi_{w_{4}}(A)=\varphi_{w_{4}}(A) w_{4}$, that is, $\varphi_{w_{4}}(A) \in\left(E_{8}\right)^{w_{4}}$. Thus $\varphi_{w_{4}}$ is well-defined. Obviously $\varphi_{w_{4}}$ is a homomorphism. Here we easily obtain $\operatorname{Ker} \varphi_{w_{4}}=\left\{E, \omega E, \omega^{2} E\right\}$, where $\omega \in C, \omega^{3}=1, \omega \neq 1$. Indeed, it follows that

$$
\begin{aligned}
& \operatorname{Ker} \varphi_{w_{4}}=\left\{A \in S(U(1) \times U(8)) \mid \varphi_{w_{4}}(A)=1\right\} \\
& =\left\{\begin{array}{l|l}
A \in S(U(1) \times U(8)) & \begin{array}{l}
A D A^{-1}=D, A \boldsymbol{u}=\boldsymbol{u},{ }^{t} A^{-1} \boldsymbol{v}=\boldsymbol{v}, \\
\text { for all } D \in \mathfrak{s l}(9, C), \boldsymbol{u}, \boldsymbol{v} \in \Lambda^{3}\left(C^{9}\right)
\end{array}
\end{array}\right\} \\
& =\left\{\begin{array}{l|l}
A \in S(U(1) \times U(8)) & \begin{array}{l}
A D=D A, A \boldsymbol{u}=\boldsymbol{u},(\tau A) \boldsymbol{v}=\boldsymbol{v}, \\
\text { for all } D \in \mathfrak{s l}(9, C), \boldsymbol{u}, \boldsymbol{v} \in \Lambda^{3}\left(C^{9}\right)
\end{array}
\end{array}\right\} \\
& =\left\{E, \omega E, \omega^{2} E\right\} \cong Z_{3} \text {. }
\end{aligned}
$$


Finally, we will prove that $\varphi_{w_{4}}$ is surjective. Since $\operatorname{Ker} \varphi_{w_{4}}$ is discrete and $\left(E_{8}\right)^{w_{4}}$ is connected, and together with $\operatorname{dim}\left(\left(\mathrm{e}_{8}\right)^{w_{4}}\right)=64=(64+1)-1=\operatorname{dim}(\mathfrak{s}(\mathfrak{u}(1) \oplus \mathfrak{u}(8)))$ (Lemma 4.9), we see that $\varphi_{w_{4}}$ is surjective. Thus we have the following isomorphism

$$
\left(E_{8}\right)^{w_{4}} \cong S(U(1) \times U(8)) / Z_{3} .
$$

Further, the mapping $f: U(1) \times S U(8) \rightarrow S(U(1) \times U(8))$,

$$
f(b, B)=\left(\begin{array}{cc}
b^{-8} & 0 \\
0 & b B
\end{array}\right),
$$

induces the isomorphism $S(U(1) \times U(8)) \cong(U(1) \times S U(8)) / \boldsymbol{Z}_{8}, \boldsymbol{Z}_{8}=\left\{\left(\omega_{8}{ }^{k}, \omega_{8}{ }^{-k} E\right) \mid k\right.$ $=0,1, \ldots, 7\}$, where $\omega_{8}=e^{i 2 \pi / 8} \in U(1)$.

Here, we will determine the kernel of the mapping $\varphi_{w_{4}, f}$ as the composition of the mappings $w_{4}$ and $f$ :

$$
\varphi_{w_{4}, f}: U(1) \times S U(8) \rightarrow S(U(1) \times U(8)) \rightarrow\left(E_{8}\right)^{w_{4}} .
$$

Using the result of $\operatorname{Ker} f$, by doing straightforward computation we have the following $\begin{aligned} \operatorname{Ker} \varphi_{w_{4}, f} & =\left\{\left(\omega_{24}{ }^{3 k}, \omega_{24}{ }^{-3 k} E\right),\left(\omega_{24}{ }^{3 k+1}, \omega_{24}{ }^{-3(k-5)} E\right),\left(\omega_{24}{ }^{3 k+2}, \omega_{24}{ }^{-3(k-2)} E\right) \mid k=0, \ldots, 7\right\} \\ & \simeq Z_{24}\end{aligned}$

Therefore, we have the desired isomorphism

$$
\left(E_{8}\right)^{w_{4}} \cong(U(1) \times S U(8)) / Z_{24} .
$$

\section{Case 3. The automorphism $\tilde{v}_{4}$ Of Order four And the group $\left(E_{8}\right)^{v_{4}}$}

In this section (also in the next Section 6), again we use the 248-dimensional vector space $\mathrm{e}_{8}{ }^{C}$ used in Case $1([\overline{5}])$ and the connected compact exceptional Lie group of type $E_{8}$ constructed by T. Imai and I. Yokota ([1]).

We define a $C$-linear transformation $v_{4}$ of $\mathrm{e}_{8}{ }^{C}$ by

$$
v_{4}(\Phi, P, Q, r, s, t)=(\Phi, i P,-i Q, r,-s,-t) .
$$

Then we see that $v_{4} \in E_{8}$ and $\left(v_{4}\right)^{4}=1,\left(v_{4}\right)^{2}=v$, where $v$ is the $C$-linear transformation of $\mathrm{e}_{8}{ }^{C}$ defined in [10, Definition of Subsection 5.7(p. 174)], and so $v_{4}$ induces the inner automorphism $\tilde{v}_{4}$ of order four on $E_{8}: \tilde{v}_{4}(\alpha)=v_{4} \alpha v_{4}{ }^{-1}, \alpha \in E_{8}$.

Now, we will study the subgroup $\left(E_{8}\right)^{v_{4}}$ of $E_{8}$ :

$$
\left(E_{8}\right)^{v_{4}}=\left\{\alpha \in E_{8} \mid v_{4} \alpha=\alpha v_{4}\right\} .
$$

The aim of the rest of this section is to determine the structure of the group $\left(E_{8}\right)^{v_{4}}$. Before that, we prove lemma and proposition needed later.

Lemma 5.1. The Lie algebra $\left(\mathrm{e}_{8}\right)^{v_{4}}$ of the group $\left(E_{8}\right)^{v_{4}}$ is given by

$$
\begin{aligned}
\left(\mathfrak{e}_{8}\right)^{v_{4}} & =\left\{R \in \mathfrak{e}_{8} \mid v_{4} R=R\right\} \\
& =\left\{R=(\Phi, 0,0, r, 0,0) \mid \Phi \in \mathfrak{e}_{7}, r \in i \boldsymbol{R}\right\} .
\end{aligned}
$$


Proof. By doing straightforward computation, we can prove this lemma.

Let $U(1)=\{\theta \in C \mid(\tau \theta) \theta=1\}$ be the unitary group. Then the ordinary unitary group $U(1)$ is isomorphic to the group $\left\{\left(\begin{array}{cc}\theta & 0 \\ 0 & \tau \theta\end{array}\right) \mid \theta \in U(1)\right\}$ as the subgroup of the group $S U(2)$. Hereafter, we denote an element $\left(\begin{array}{cc}\theta & 0 \\ 0 & \tau \theta\end{array}\right)$ by $A_{\theta}: A_{\theta}=\left(\begin{array}{cc}\theta & 0 \\ 0 & \tau \theta\end{array}\right)$.

Proposition 5.2. The group $\left(E_{8}\right)^{v_{4}}$ contains a subgroup

$$
\phi_{v}(U(1))=\left\{\phi_{v}\left(A_{\theta}\right) \in E_{8} \mid A_{\theta} \in U(1)\right\}
$$

which is isomorphic to the group $U(1)=\{\theta \in C \mid(\tau \theta) \theta=1\}$, where $\phi_{v}$ is same one as $\varphi_{3}$ defined in [10, Theorem 5.7.4], and moreover the explicit form of $\phi_{v}\left(A_{\theta}\right): \mathrm{e}_{8} C \rightarrow \mathrm{e}_{8}{ }^{C}$ is given as follows:

$$
\phi_{v}\left(A_{\theta}\right)(\Phi, P, Q, r, s, t)=\left(\Phi, \theta P,(\tau \theta) Q, r, \theta^{2} s,(\tau \theta)^{2} t\right) .
$$

In particular, we have $v_{4}=\phi_{v}\left(A_{i}\right), i \in U(1)$.

Proof. For $A_{\theta}=\left(\begin{array}{cc}\theta & 0 \\ 0 & \tau \theta\end{array}\right)=\exp \left(\begin{array}{cc}-i v & 0 \\ 0 & i \nu\end{array}\right) \in U(1) \subset S U(2)$, by Lemma 5.1 and [10, Theorem 5.7.4] we have $\phi_{v}\left(A_{\theta}\right)=\exp (\operatorname{ad}(0,0,0, i v, 0,0)) \in\left(E_{8}\right)^{v_{4}}$, moreover the explicit form of $\phi_{v}\left(A_{\theta}\right)$ and the result of $v_{4}=\phi_{v}\left(A_{i}\right)$ are the direct results from [10, Theorem 5.7.4]).

Here, let $E_{7}$ be the compact connected exceptional Lie group of type $E_{7}$ ([10, Definition of Subsection 4.2 (p.108)]), then we confirm that $\alpha \in E_{7}$ satisfies $\alpha 1=$ $1, \alpha 1^{-}=1^{-}$and $\alpha 1_{-}=1_{-}$, where $1=(0,0,0,1,0,0), 1^{-}=(0,0,0,0,1,0), 1_{-}=$ $(0,0,0,0,0,1) \in \mathfrak{e}_{8}{ }^{C}$ (see [10, Lemma 5.7.2, Theorem 5.7.3] for details).

Now, we will determine the structure of the group $\left(E_{8}\right)^{v_{4}}$.

Theorem 5.3. The group $\left(E_{8}\right)^{v_{4}}$ is isomorphic to the group $\left(U(1) \times E_{7}\right) / \boldsymbol{Z}_{2}, \boldsymbol{Z}_{2}=\{(1,1)$, $(-1,-1)\}:\left(E_{8}\right)^{v_{4}} \cong\left(U(1) \times E_{7}\right) / Z_{2}$.

Proof. We define a mapping $\varphi_{v_{4}}: U(1) \times E_{7} \rightarrow\left(E_{8}\right)^{v_{4}}$ by

$$
\varphi_{v_{4}}(\theta, \delta)=\phi_{v}\left(A_{\theta}\right) \delta \text {. }
$$

We will prove that $\varphi_{v_{4}}$ is well- defined. First, we can confirm that the group $E_{7}$ is the subgroup of the group $\left(E_{8}\right)^{v_{4}}$. Indeed, let $\delta \in E_{7}$, then it follows that

$$
\begin{aligned}
v_{4} \delta(\Phi, P, Q, r, s, t) & =v_{4}\left(\delta \Phi \delta^{-1}, \delta P, \delta Q, r, s, t\right), \\
& =\left(\delta \Phi \delta^{-1}, i \delta P,-i \delta Q, r,-s,-t\right) \\
& =\left(\delta \Phi \delta^{-1}, \delta(i P), \delta(-i Q), r,-s,-t\right) \\
& =\delta v_{4}(\Phi, P, Q, r, s, t),(\Phi, P, Q, r, s, t) \in \mathrm{e}_{8}{ }^{C},
\end{aligned}
$$


that is, $v_{4} \delta=\delta v_{4}$. Hence we have $E_{7} \subset\left(E_{8}\right)^{v_{4}}$, so together with Proposition 5.2, we see that $\varphi_{v_{4}}$ is well-defined. Subsequently, since the mapping $\varphi_{v_{4}}$ is the restriction mapping of $\varphi_{v}: S U(2) \times E_{7} \rightarrow\left(E_{8}\right)^{v}$ ([10, Theorem 5.7.6]), $\varphi_{v_{4}}$ is a homomorphism.

Next, we will prove that $\varphi_{v_{4}}$ is surjective. Let $\alpha \in\left(E_{8}\right)^{v_{4}} \subset\left(E_{8}\right)^{v}$. Then there exist $A \in S U(2)$ and $\delta \in E_{7}$ such that $\alpha=\varphi_{v}(A, \delta)$. Moreover, from the condition $v_{4} \alpha v_{4}^{-1}=\alpha$, that is, $v_{4} \varphi_{v}(A, \delta) v_{4}^{-1}=\varphi_{v}(A, \delta)$, as $A=\left(\begin{array}{ll}a & b \\ c & d\end{array}\right) \in S U(2)$, this relational formula is expressed as follows:

$$
\varphi_{v}\left(\left(\begin{array}{cc}
a & -b \\
-c & d
\end{array}\right), v_{4} \delta v_{4}^{-1}\right)=\varphi_{v}\left(\left(\begin{array}{ll}
a & b \\
c & d
\end{array}\right), \delta\right) .
$$

Indeed, from Proposition 5.2, we easily see

$$
v_{4} \phi_{v}(A) v_{4}^{-1}=\phi_{v}\left(\left(\begin{array}{cc}
i & 0 \\
0 & -i
\end{array}\right)\right) \phi_{v}\left(\left(\begin{array}{ll}
a & b \\
c & d
\end{array}\right)\right) \phi_{v}\left(\left(\begin{array}{cc}
-i & 0 \\
0 & i
\end{array}\right)\right)=\phi_{v}\left(\left(\begin{array}{cc}
a & -b \\
-c & d
\end{array}\right)\right),
$$

and so it follows that

$$
\begin{aligned}
v_{4} \varphi_{v}(A, \delta) v_{4}^{-1} & =v_{4}\left(\phi_{v}(A) \delta\right) v_{4}^{-1}=\left(v_{4} \phi_{v}(A) v_{4}^{-1}\right)\left(v_{4} \delta v_{4}^{-1}\right) \\
& =\phi_{v}\left(\left(\begin{array}{cc}
a & -b \\
-c & d
\end{array}\right)\right)\left(v_{4} \delta v_{4}^{-1}\right) \\
& =\varphi_{v}\left(\left(\begin{array}{cc}
a & -b \\
-c & d
\end{array}\right), v_{4} \delta v_{4}^{-1}\right) .
\end{aligned}
$$

Thus we have the following

$$
\left\{\begin{array} { l } 
{ ( \begin{array} { c c } 
{ a } & { - b } \\
{ - c } & { d }
\end{array} ) = ( \begin{array} { l l } 
{ a } & { b } \\
{ c } & { d }
\end{array} ) } \\
{ v _ { 4 } \delta v _ { 4 } ^ { - 1 } = \delta }
\end{array} \quad \text { or } \quad \left\{\begin{array}{l}
\left(\begin{array}{cc}
a & -b \\
-c & d
\end{array}\right)=-\left(\begin{array}{ll}
a & b \\
c & d
\end{array}\right) \\
v_{4} \delta v_{4}^{-1}=-\delta .
\end{array}\right.\right.
$$

In the former case, from the first condition we have $A=\operatorname{diag}(a, \tau a), a \in U(1)$. Since $\delta \in E_{7}$ leaves the condition $v_{4} \delta v_{4}^{-1}=\delta$ as mentioned in the beginning of this proof, it is trivial $\delta \in E_{7}$. Hence there exist $\theta \in U(1)$ and $\delta \in E_{7}$ such that $\alpha=\varphi_{v}\left(A_{\theta}, \delta\right)=$ $\varphi_{v_{4}}(\theta, \delta)$. In the latter case, since we have $\delta=-\delta$ from the second condition, that is, $\delta=0$, this case is impossible. The proof of surjective is completed.

Finally, from $\operatorname{Ker} \varphi_{v}=\{(E, 1),(-E,-1)\}$, we easily obtain $\operatorname{Ker} \varphi_{v_{4}}=\{(E, 1),(-E,-1)\}$ $\cong Z_{2}$.

Therefore, we have the desired isomorphism

$$
\left(E_{8}\right)^{v_{4}} \cong\left(U(1) \times E_{7}\right) / Z_{2}
$$

\section{Case 4. The automorphism $\tilde{\mu}_{4}$ OF ORder four ANd the Group $\left(E_{8}\right)^{\mu_{4}}$}

We define $C$-linear transformations of $\mathfrak{P}^{C}$ by

$$
\lambda(X, Y, \xi, \eta)=(Y,-X, \eta,-\xi), \quad \gamma(X, Y, \xi, \eta)=(\gamma X, \gamma Y, \xi, \eta),
$$


where $\gamma$ on the right hand side is same one as $\gamma \in G_{2} \subset F_{4} \subset E_{6}$ (see [10, Subsections 1.10(p.19), 2.11(p.59), 3.11(p.90)] for details) and $\mathfrak{P}^{C}$ is the Freudenthal $C$-vector space (see [10, Section 2 (p.94)]). Then we have $\lambda \in E_{7}, \lambda^{4}=1, \lambda^{2}=-1$ ([10, Lemma 4.3.3]) and $\gamma \in E_{7}, \gamma^{2}=1$.

Using these transformations, we define a $C$-linear transformation $\mu_{4}$ of $\mathrm{e}_{8}{ }^{C}$ by

$$
\mu_{4}(\Phi, P, Q, r, s, t)=\left(\lambda \gamma \Phi \gamma \lambda^{-1},-\lambda \gamma P,-\lambda \gamma Q, r, s, t\right) .
$$

Then we see $\mu_{4} \in E_{8}$ and $\left(\mu_{4}\right)^{4}=1,\left(\mu_{4}\right)^{2}=v$, where $v$ is same one in previous section, and so $\mu_{4}$ induces the inner automorphism $\tilde{\mu}_{4}$ of order four on $E_{8}: \tilde{\mu}_{4}(\alpha)=$ $\mu_{4} \alpha \mu_{4}^{-1}, \alpha \in E_{8}$.

Now, we will study the subgroup $\left(E_{8}\right)^{\mu_{4}}$ of $E_{8}$ :

$$
\left(E_{8}\right)^{\mu_{4}}=\left\{\alpha \in E_{8} \mid \mu_{4} \alpha=\alpha \mu_{4}\right\} .
$$

The aim of the rest of this section is to determine the structure of the group $\left(E_{8}\right)^{\mu_{4}}$. Before that, we prove lemma and propositions needed later.

Lemma 6.1. The Lie algebra $\left(\mathrm{e}_{8}\right)^{\mu_{4}}$ of the group $\left(E_{8}\right)^{\mu_{4}}$ is given by

$$
\begin{aligned}
\left(\mathfrak{e}_{8}\right)^{\mu_{4}} & =\left\{R \in \mathfrak{e}_{8} \mid \mu_{4} R=R\right\} \\
& =\left\{R=(\Phi, 0,0, r, s,-\tau s) \mid \Phi \in\left(\mathfrak{e}_{7}\right)^{\lambda \gamma}, r \in i \boldsymbol{R}, s \in C\right\} .
\end{aligned}
$$

Proof. By doing straightforward computation, we can prove this lemma.

Proposition 6.2. The group $\left(E_{8}\right)^{\mu_{4}}$ contains a subgroup

$$
\phi_{v}(S U(2))=\left\{\phi_{v}(A) \in E_{8} \mid A \in S U(2)\right\}
$$

which is isomorphic to the group $S U(2)=\left\{A \in M(2, C) \mid\left(\tau^{t} A\right) A=E, \operatorname{det} A=1\right\}$, where $\phi_{v}$ is defined in Proposition 5.2.

Proof. As in the proof of Proposition 5.2 for $A=\left(\begin{array}{cc}a & -\tau b \\ b & \tau a\end{array}\right)=\exp \left(\begin{array}{cc}-i v & -\tau \rho \\ \rho & i v\end{array}\right) \in$ $S U(2)$, by Lemma 6.1 and [9, Theorem 5.7.4] we have $\phi_{v}(A)=\exp (\operatorname{ad}(0,0,0, i v$, $\rho,-\tau \rho)) \in\left(E_{8}\right)^{\mu_{4}}$.

Here, again let $E_{7}$ be the compact connected exceptional Lie group of type $E_{7}$, we consider the following subgroup $\left(E_{7}\right)^{\lambda \gamma}$ of $E_{7}$ :

$$
\left(E_{7}\right)^{\lambda \gamma}=\left\{\alpha \in E_{7} \mid(\lambda \gamma) \alpha\left(\gamma \lambda^{-1}\right)=\alpha\right\} .
$$

Then we have the following proposition.

Proposition 6.3. The group $\left(E_{7}\right)^{\lambda \gamma}$ is the subgroup of the group $\left(E_{8}\right)^{\mu_{4}}:\left(E_{7}\right)^{\lambda \gamma} \subset\left(E_{8}\right)^{\mu_{4}}$.

Proof. Let $\alpha \in\left(E_{7}\right)^{\lambda \gamma}$. Note that $-1 \in z\left(E_{7}\right)$ (the center of $E_{7}$ ), we have the following

$$
\begin{aligned}
\mu_{4} \alpha(\Phi, P, Q, r, s, t) & =\mu_{4}\left(\alpha \Phi \alpha^{-1}, \alpha P, \alpha Q, r, s, t\right) \\
& =\left(\lambda \gamma \alpha \Phi \alpha^{-1} \gamma \lambda^{-1},-\lambda \gamma \alpha P,-\lambda \gamma \alpha Q, r, s, t\right) \\
& =\left(\alpha\left(\lambda \gamma \Phi \gamma \lambda^{-1}\right) \alpha^{-1}, \alpha(-\lambda \gamma P), \alpha(-\lambda \gamma Q), r, s, t\right)
\end{aligned}
$$




$$
=\alpha \mu_{4}(\Phi, P, Q, r, s, t),(\Phi, P, Q, r, s, t) \in \mathrm{e}_{8}{ }^{C},
$$

that is, $\mu_{4} \alpha=\alpha \mu_{4}$. Hence we have the required result $\left(E_{7}\right)^{\lambda \gamma} \subset\left(E_{8}\right)^{\mu_{4}}$.

Now, we will determine the structure of the group $\left(E_{8}\right)^{\mu_{4}}$.

Theorem 6.4. The group $\left(E_{8}\right)^{\mu_{4}}$ is isomorphic to the group $(S U(2) \times S U(8)) / \boldsymbol{Z}_{4}, \boldsymbol{Z}_{4}=$ $\left\{(E, E),(E,-E),\left(-E, e_{1} E\right),\left(-E,-e_{1} E\right)\right\}:\left(E_{8}\right)^{\mu_{4}} \cong(S U(2) \times S U(8)) / Z_{4}$.

Proof. We define a mapping $\varphi_{\mu_{4}}: S U(2) \times S U(8) \rightarrow\left(E_{8}\right)^{\mu_{4}}$ by

$$
\varphi_{\mu_{4}}(A, L)=\phi_{v}(A) \varphi_{S U(8)}(L),
$$

where the mapping $\varphi_{S U(8)}: S U(8) \rightarrow\left(E_{7}\right)^{\tau \gamma}=\left(E_{7}\right)^{\lambda \gamma} \subset E_{7}$ is same one as $\varphi$ defined in [10, Theorem 4.12.5]. From Propositions 6.2, 6.3, we see that $\varphi_{\mu_{4}}$ is well-defined. Subsequently, as in the proof of Theorem 5.3. Since the mapping $\varphi_{\mu_{4}}$ is the restriction mapping of $\varphi_{v}: S U(2) \times E_{7} \rightarrow\left(E_{8}\right)^{v}$ ([10, Theorem 5.7.6]), $\varphi_{\mu_{4}}$ is a homomorphism.

Next, we will prove that $\varphi_{\mu_{4}}$ is surjective. Let $\alpha \in\left(E_{8}\right)^{\mu_{4}} \subset\left(E_{8}\right)^{v}$. Then there exist $A \in S U(2)$ and $\delta \in E_{7}$ such that $\alpha=\varphi_{v}(A, \delta)$. Moreover, from the condition $\mu_{4} \alpha \mu_{4}^{-1}=\alpha$, that is, $\mu_{4} \varphi_{v}(A, \delta) \mu_{4}^{-1}=\varphi_{v}(A, \delta)$, this relational formula is expressed by

$$
\varphi_{v}\left(A,(\lambda \gamma) \delta\left(\gamma \lambda^{-1}\right)\right)=\varphi_{v}(A, \delta)
$$

Indeed, from $\mu_{4}=\mu \lambda \gamma$ and [9, Lemma 5.4.3] we easily see $\mu_{4} \phi_{v}(A) \mu_{4}^{-1}=\phi_{v}(A)$, and it is clear that $\mu_{4} \delta \mu_{4}^{-1}=(\lambda \gamma) \delta\left(\gamma \lambda^{-1}\right)$ because of $\delta \in E_{7}$. Hence it follows from

$$
\begin{aligned}
\mu_{4} \varphi_{v}(A, \delta) \mu_{4}^{-1} & =\mu_{4}\left(\phi_{v}(A) \delta\right) \mu_{4}^{-1}=\left(\mu_{4} \phi_{v}(A) \mu_{4}^{-1}\right)\left(\mu_{4} \delta \mu_{4}^{-1}\right) \\
& =\phi_{v}(A)\left((\lambda \gamma) \delta\left(\gamma \lambda^{-1}\right)\right) \\
& =\varphi_{v}\left(A,(\lambda \gamma) \delta\left(\gamma \lambda^{-1}\right)\right)
\end{aligned}
$$

that $\varphi_{v}\left(A,(\lambda \gamma) \delta\left(\gamma \lambda^{-1}\right)\right)=\varphi_{v}(A, \delta)$.

Thus we have the following

$$
\left\{\begin{array} { l } 
{ A = A } \\
{ ( \lambda \gamma ) \delta ( \gamma \lambda ^ { - 1 } ) = \delta }
\end{array} \quad \text { or } \quad \left\{\begin{array}{l}
A=-A \\
(\lambda \gamma) \delta\left(\gamma \lambda^{-1}\right)=-\delta .
\end{array}\right.\right.
$$

In the latter case, this case is impossible because of $A \neq 0$. In the former case, it is clear $A \in S U(2)$, as for the second condition, there exists $L \in S U(8)$ such that $\delta=\varphi_{S U(8)}(L)$ from [10, Theorem 4.12.5]. Hence there exist $A \in S U(2)$ and $L \in S U(8)$ such that $\alpha=\varphi_{v}\left(A, \varphi_{S U(8)}(L)\right)=\varphi_{\mu_{4}}(A, L)$. The proof of surjective is completed.

Finally, it is not difficult to obtain $\operatorname{Ker} \varphi_{\mu_{4}}=\left\{(E, E),(E,-E),\left(-E, e_{1} E\right),\left(-E,-e_{1} E\right)\right\}$ $\cong Z_{4}$, where $e_{1}$ is one of basis in $\mathfrak{C}$. Indeed, first it follows from the definition of kernel that

$$
\begin{aligned}
\operatorname{Ker} \varphi_{\mu_{4}} & =\left\{(A, L) \in S U(2) \times S U(8) \mid \varphi_{\mu_{4}}(A, L)=1\right\} \\
& =\left\{(A, L) \in S U(2) \times S U(8) \mid \phi_{v}(A) \varphi_{S U(8)}(L)=1\right\} .
\end{aligned}
$$


Here, since the mapping $\varphi_{\mu_{4}}$ is the restriction of the mapping $\varphi_{v}: S U(2) \times E_{7} \rightarrow\left(E_{8}\right)^{v}$, it follows from $\operatorname{Ker} \varphi_{v}=\{(E, 1),(-E,-1)\}([10$, Theorem 5.7.6]) that

$$
\begin{aligned}
\operatorname{Ker} \varphi_{\mu_{4}} & =\left\{(A, L) \in S U(2) \times S U(8) \mid A=E, \varphi_{S U(8)}(L)=1\right\} \\
& \cup\left\{(A, L) \in S U(2) \times S U(8) \mid A=-E, \varphi_{S U(8)}(L)=-1\right\} .
\end{aligned}
$$

In the former case, from $\operatorname{Ker} \varphi_{S U(8)}=\{E,-E\}$, we have the following

$$
\left\{\begin{array} { l } 
{ A = E } \\
{ L = E }
\end{array} \quad \text { or } \quad \left\{\begin{array}{l}
A=E \\
L=-E .
\end{array}\right.\right.
$$

In the latter case, using $-1=\varphi_{S U(8)}\left(e_{1} E\right)=\varphi_{S U(8)}\left(-e_{1} E\right)$, we have the following

$$
\left\{\begin{array} { l } 
{ A = - E } \\
{ L = e _ { 1 } E }
\end{array} \quad \text { or } \quad \left\{\begin{array}{l}
A=-E \\
L=-e_{1} E .
\end{array}\right.\right.
$$

Hence we have the required result

$$
\operatorname{Ker} \varphi_{\mu_{4}}=\left\{(E, E),(E,-E),\left(-E, e_{1} E\right),\left(-E,-e_{1} E\right)\right\} \cong Z_{4} .
$$

Therefore, we have the desired isomorphism

$$
\left(E_{8}\right)^{\mu_{4}} \cong(S U(2) \times S U(8)) / \boldsymbol{Z}_{4} .
$$

\section{REFERENCES}

[1] T. Imai and I. Yokota, Simply connected compact simple Lie group $E_{8(-248)}$ of type $E_{8}$, J. Math. Kyoto Univ. 21(1981), 741-762.

[2] S. Gomyo, Realization of maximal subgroups of rank 8 of the simply connected compact simple Lie group of type $E_{8}$, Tsukuba J. Math. 21(1997), 595-616.

[3] J.A. Jiménez, Riemannian 4-symmetric spaces, Trans. Amer. Math. Soc. 306 (1988), 715-734.

[4] T. Miyashita, Realizations of globally exceptional $Z_{2} \times Z_{2}$-symmetric spaces, Tsukuba J. Math. 38-2(2014), 239-311.

[5] T. Miyashita, Realizations of inner automorphisms of order 4 and fixed points subgroups by them on the connected compact exceptional Lie group $E_{8}$, Part I, Tsukuba J. Math. 41-1(2017), 91-166.

[6] T. Miyashita, Realizations of inner automorphisms of order four and fixed points subgroups by them on the connected compact exceptional Lie group $E_{8}$, Part III, in preparation.

[7] I. Yokota, Realizations of involutive automorphisms $\sigma$ and $G^{\sigma}$ of exceptional linear Lie groups $G$, Part I, $G=G_{2}, F_{4}$, and $E_{6}$, Tsukuba J. Math. 14(1990), 185-223.

[8] I. Yokota, Realizations of involutive automorphisms $\sigma$ and $G^{\sigma}$ of exceptional linear Lie groups $G$, Part II, $G=E_{7}$, Tsukuba J. Math. 14(1990), 378-404.

[9] I. Yokota, Realizations of involutive automorphisms $\sigma$ and $G^{\sigma}$ of exceptional linear Lie groups $G$, Part III, $G=E_{8}$, Tsukuba J. Math. 15(1991), 301-314.

[10] I. Yokota, Exceptional Lie groups, arXiv:math/0902.0431vl[mathDG] (2009).

1365-3 BESSHO ONSEN

Ueda City

Nagano Prefecture 386-1431

JAPAN

E-mail address: anarchybin@gmail.com 